\title{
Temperature retrieval from Rayleigh-Brillouin scattering profiles measured in air
}

\author{
Benjamin Witschas, ${ }^{1, *}$ Ziyu Gu, ${ }^{2}$ and Wim Ubachs ${ }^{2}$ \\ ${ }^{1}$ Deutsches Zentrum für Luft- und Raumfahrt (DLR), Institut für Physik der Atmosphäre, \\ Oberpfaffenhofen 82234, Germany \\ ${ }^{2}$ Department of Physics and Astronomy, LaserLaB, VU University, De Boelelaan 1081, 1081 \\ HV Amsterdam, Netherlands \\ *Benjamin.Witschas@dlr.de
}

\begin{abstract}
In order to investigate the performance of two different algorithms for retrieving temperature from Rayleigh-Brillouin (RB) line shapes, $\mathrm{RB}$ scattering measurements have been performed in air at a wavelength of $403 \mathrm{~nm}$, for a temperature range from $257 \mathrm{~K}$ to $330 \mathrm{~K}$, and atmospherically relevant pressures from $871 \mathrm{hPa}$ to $1013 \mathrm{hPa}$. One algorithm, based on the Tenti S6 line shape model, shows very good accordance with the reference temperature. In particular, the absolute difference is always less than $2 \mathrm{~K}$. A linear correlation yields a slope of $1.01 \pm 0.02$ and thus clearly demonstrates the reliability of the retrieval procedure. The second algorithm, based on an analytical line shape model, shows larger discrepancies of up to $9.9 \mathrm{~K}$ and is thus not useful at its present stage. The possible reasons for these discrepancies and improvements of the analytical model are discussed. The obtained outcomes are additionally verified with previously performed $\mathrm{RB}$ measurements in air, at $366 \mathrm{~nm}$, temperatures from $255 \mathrm{~K}$ to $338 \mathrm{~K}$ and pressures from $643 \mathrm{hPa}$ to $826 \mathrm{hPa}$ [Appl. Opt. 52, 4640 (2013)]. The presented results are of relevance for future lidar studies that might utilize RB scattering for retrieving atmospheric temperature profiles with high accuracy.
\end{abstract}

(C) 2014 Optical Society of America

OCIS codes: (010.0010) Atmospheric and oceanic optics; (280.0280) Remote sensing and sensors; (300.0300) Spectroscopy; (280.3640) Lidar; (280.1310) Atmospheric scattering; (290.5820) Scattering measurements; (300.6390) Spectroscopy, molecular.

\footnotetext{
References and links

1. M. Alpers, R. Eixmann, C. Fricke-Begemann, M. Greding, and J. Höffner, "Temperature lidar measurements from 1 to $105 \mathrm{~km}$ altitude using resonance, Rayleigh, and Rotational Raman scattering," Atmos. Chem. Phys. 4, $793-800$ (2004).

2. A. Behrendt and J. Reichardt, "Atmospheric temperature profiling in the presence of clouds with a pure rotational Raman lidar by use of an interference-filter-based polychromator," Appl. Opt. 39, 1372-1378 (2000).

3. A. Behrendt, T. Nakamura, and T. Tsuda, "Combined temperature lidar for measurements in the troposphere, stratosphere, and mesosphere," Appl. Opt. 43, 2930-2939 (2004).

4. M. Radlach, A. Behrendt, and V. Wulfmeyer, "Scanning rotational Raman lidar at $355 \mathrm{~nm}$ for the measurement of tropospheric temperature fields," Atmos. Chem. Phys. 8, 159-169 (2008).

5. B. Witschas, "Light scattering on molecules in the atmosphere," in Atmospheric Physics: Background - Methods - Trends, U. Schumann, ed. (Springer, 2012), pp. 69-83.

6. G. Fiocco, G. Benedetti-Michelangeli, K. Maischberger, and E. Madonna, "Measurement of temperature and aerosol to molecule ratio in the troposphere by optical radar," Nat. Phys. Sci. 229, 78-79 (1971).
}

\#221756 - \$15.00 USD Received 26 Aug 2014; revised 9 Oct 2014; accepted 9 Oct 2014; published 19 Nov 2014

(C) 2014 OSA 1 December $2014 \mid$ Vol. 22, No. 24 | DOI:10.1364/OE.22.029655 | OPTICS EXPRESS 29655 
7. A. Young and G. Kattawar, "Rayleigh-scattering line profiles," Appl. Opt. 22, 3668-3670 (1983).

8. G. Tenti, C. Boley, and R. Desai, "On the kinetic model description of Rayleigh-Brillouin scattering from molecular gases," Can. J. Phys. 52, 285-290 (1974).

9. B. Witschas, M. O. Vieitez, E. J. van Duijn, O. Reitebuch, W. van de Water, and W. Ubachs, "Spontaneous Rayleigh-Brillouin scattering of ultraviolet light in nitrogen, dry air, and moist air," Appl. Opt. 49, 4217-4227 (2010).

10. M. O. Vieitez, E. J. van Duijn, W. Ubachs, B. Witschas, A. Meijer, A. S. de Wijn, N. Dam, and W. van de Water, "Coherent and spontaneous Rayleigh-Brillouin scattering in atomic and molecular gases and gas mixtures," Phys. Rev. A 82, 1094-1622 (2010).

11. Z. Y. Gu, B. Witschas, W. van de Water, and W. Ubachs, "Rayleigh-Brillouin scattering profiles of air at different temperatures and pressures," Appl. Opt. 52, 4640-4651 (2013).

12. Z. Y. Gu and W. Ubachs, "A systematic study of Rayleigh-Brillouin scattering in air, $\mathrm{N}_{2}$, and $\mathrm{O}_{2}$ gases," J. Chem. Phys. 141, 104329 (2014).

13. B. Witschas, "Analytical model for Rayleigh-Brillouin line shapes in air," Appl. Opt. 50, 267-270 (2011).

14. B. Witschas, "Analytical model for Rayleigh-Brillouin line shapes in air: errata," Appl. Opt. 50, 5758 (2011).

15. Z. Y. Gu, M. O. Vieitez, E. J. van Duijn, and W. Ubachs, "A Rayleigh-Brillouin scattering spectrometer for ultraviolet wavelengths,” Rev. Sci. Instrum. 83, 053112 (2012).

16. P. Wilksch, "Instrument function of the Fabry-Perot spectrometer," Appl. Opt. 24, 1502-1511 (1985).

17. M. McGill, W. Skinner, and T. Irgang, "Analysis techniques for the recovery of winds and backscatter coefficients from a multiple-channel incoherent Doppler lidar," Appl. Opt. 36, 1253-1268 (1997).

18. B. Witschas, C. Lemmerz, and O. Reitebuch, "Horizontal lidar measurements for the proof of spontaneous Rayleigh-Brillouin scattering in the atmosphere," Appl. Opt. 51, 6207-6219 (2012).

19. T. D. Rossing (Ed.), Springer Handbook of Acoustics (Springer, 2007).

20. N. Hagen, M. Kupinski, and E. L. Dereniak, "Gaussian profile estimation in one dimension," Appl. Opt. 46. 5374-5383 (2007).

21. Y. Ma, F. Fan, K. Liang, H. Li, Y. Yu, and B. Zhou, "An analytical model for Rayleigh-Brillouin scattering spectra in gases," J. Opt. 14, 095703 (2012).

22. Y. Ma, H. Li, Z. Y. Gu, W. Ubachs, Y. Yu, J. Huang, B. Zhou, Y. Wang, and K. Liang, "Analysis of RayleighBrillouin spectral profiles and Brillouin shifts in nitrogen gas and air," Opt. Express 22, 2092-2104 (2014).

23. B. Witschas, C. Lemmerz, and O. Reitebuch, "Daytime measurements of atmospheric temperature profiles (215 km) by lidar utilizing Rayleigh-Brillouin scattering," Opt. Lett. 39, 1972-1975 (2014).

\section{Introduction}

Temperature measurements in the Earth atmosphere are important both as input for atmospheric models and as input for retrievals of other atmospheric properties such as wind, relative humidity or trace gas concentrations. Currently, lidar (light detection and ranging) instruments enable the measurement of temperature with high accuracy $(\approx 1 \mathrm{~K})$, high resolution $(\approx 100 \mathrm{~m})$ and long range (from ground up to $105 \mathrm{~km}$ ) [1]. For temperature profiling between $0 \mathrm{~km}$ and $25 \mathrm{~km}$ it is common to make use of rotational Raman scattering on air molecules, whereby the temperature dependence of the intensities of rotational Raman lines is exploited [2, 3, 4]. However, although it was demonstrated that such lidars can measure temperature during daytime [4] and in the presence of clouds [2], they suffer from the low Raman scattering cross section. Thus, powerful lasers, sophisticated background filters or night-time operation are required to obtain reliable results. In particular, the rotational Raman scattering cross section (considering Stokes and anti-Stokes branches) is about a factor of 50 smaller than that of Rayleigh scattering [5]. This marks the advantage of deriving atmospheric temperature profiles from Rayleigh-Brillouin (RB) scattering requiring high spectral resolution lidars.

Temperature is a measure of the average kinetic energy of molecules which has its imprint on the RB spectrum. Thus, atmospheric temperature can be derived by resolving the RB spectrum with high spectral resolution filters as for instance atomic vapor cells or Fabry-Pérot interferometers (FPI) and relating the measured spectrum to an appropriate line shape model. The narrow RB line width of a few GHz additionally enables the application of narrow band filters to suppress solar radiation and thus allows for daytime operation. This approach was already suggested and demonstrated in 1971 by Fiocco et al. [6], although their experimental data were severely contaminated by Mie scattering.

\#221756 - \$15.00 USD Received 26 Aug 2014; revised 9 Oct 2014; accepted 9 Oct 2014; published 19 Nov 2014

(C) 2014 OSA 1 December 2014 | Vol. 22, No. 24 | DOI:10.1364/OE.22.029655 | OPTICS EXPRESS 29656 
As further discussed by Young and Kattawar in 1983 [7], the accuracy of deriving temperature from RB spectra is strongly dependent on the quality of the RB line shape model. In 1974, Tenti et al. [8] developed a model - the so-called Tenti S6 model - for describing RB spectra of light scattered in molecular gases of single species, which is since then considered as the best model available. In particular, they use macroscopic gas transport properties as shear viscosity, bulk viscosity and thermal conductivity for describing microscopic fluctuations within the scattering gas and with it the RB spectrum. However, as air is a mixture of gases, the applicability of the Tenti S6 model to air required validation. Although some laboratory studies on RB scattering have been performed in molecular gases and gas mixtures since the early 1970s, it was not before 2010 that the first RB measurements in air were published $[9,10]$. The investigations showed that the Tenti S6 model describes the measured RB spectra in air with deviations smaller than $2 \%$ considering air as an "effective" medium, consisting of molecules with an effective mass whose collisions are parametrized by effective transport coefficients. Recently, the study was extended by Gu et al. $[11,12]$ for a temperature range from $255 \mathrm{~K}$ to $340 \mathrm{~K}$, leading to the same result. Thus, the applicability of the Tenti S6 model to RB spectra measured in air was confirmed with these experiments.

In order to retrieve temperature from measured RB profiles, they have to be analyzed with the line shape model in a certain optimization procedure as for instance a least-squares fit with temperature as free fitting parameter. As for such method it is an important issue how the $2 \%$ model deviation transfers into a temperature error. So as to deal with this issue, RB scattering measurements in air at a wavelength of $403 \mathrm{~nm}$, at a scattering angle of $91.7^{\circ}$, for temperatures from $257 \mathrm{~K}$ to $330 \mathrm{~K}$ and pressures from $871 \mathrm{hPa}$ to $1013 \mathrm{hPa}$ were performed. After that, two different temperature retrieval algorithms, one based on the Tenti S6 model, and one on an analytical line shape model according to Witschas $[13,14]$, are applied to the measured RB spectra.

\section{Experimental details}

\subsection{The instrumental setup}

RB scattering is measured from a laser beam of effectively 4 Watt, due to enhancement in a cavity, and a wavelength of $403 \mathrm{~nm}$, at a bandwidth of $2 \mathrm{MHz}$. The experimental setup is schematically shown in Fig. 1, and for further details we refer to [12, 15]. Details about the respective measurement conditions are additionally summarized in Table 2.

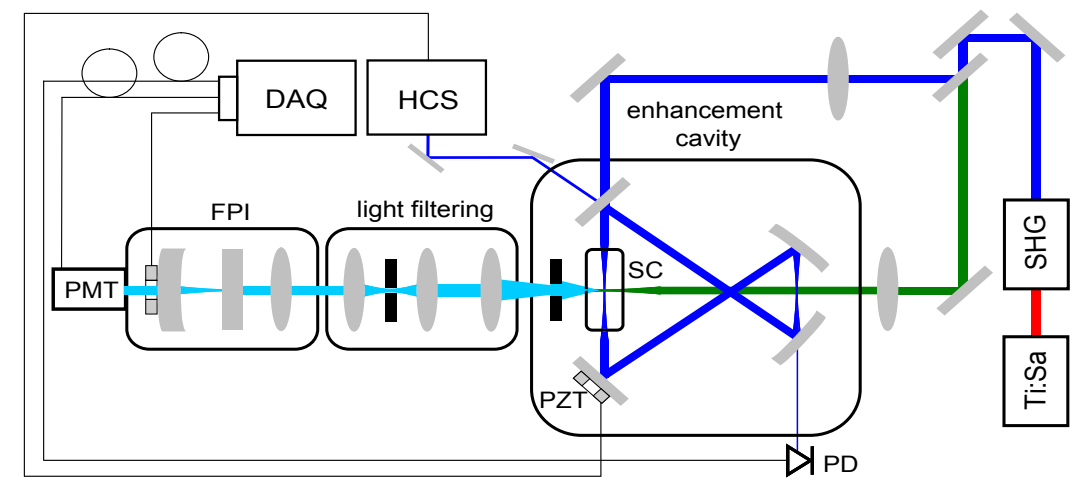

Fig. 1. Schematic diagram of the experimental setup. red: Ti:Sa laser beam $(806 \mathrm{~nm})$, dark blue: blue beam (403 nm), light blue: scattered radiation, green: reference beam, SC: scattering cell, PD: photo diode, PZT: piezo-electrical translator, FPI: Fabry-Pérot interferometer, PMT: photomultiplier, HCS: Hänsch-Couillaud stabilization, DAQ: data acquisition unit.

\#221756 - \$15.00 USD Received 26 Aug 2014; revised 9 Oct 2014; accepted 9 Oct 2014; published 19 Nov 2014

(C) 2014 OSA 1 December 2014 | Vol. 22, No. 24 | DOI:10.1364/OE.22.029655 | OPTICS EXPRESS 29657 
The light scattered in the scattering cell (Fig. 1, light blue line) is collected at an angle of $91.7^{\circ}$, with an uncertainty in a range of $\pm 1.2^{\circ}$, determined from geometrical measurement procedures employing sets of diaphragms and pinholes placed in the optical setup. This scattering angle range is later (section 3.2) used to estimate the upper limit of the uncertainty of the retrieved temperature. Considering that lidar measurements employ scattering angles of $180^{\circ}$ by definition, it would be preferable to have the same angle also for the laboratory measurements. However, as spurious scattering on optical elements within the experimental setup always lead to contamination of the measured RB line shape, it was decided to use a scattering angle different from $180^{\circ}$.

The frequency spectrum of the scattered light is resolved by means of a scanning FPI. The FPI is built as a hemispherical version of a confocal etalon, which means that it is composed of one spherical and one plane mirror. In order to scan the FPI plate distance, the spherical mirror is mounted on a piezo-electrical translator (Fig. 1, PZT) which is controlled by a computer (Fig. 1, DAQ). Despite the lower light gathering in comparison to a plane parallel FPI, the hemispherical configuration was chosen because of its insensitivity to small changes in tilt and orientation which can occur during scanning. The light that passes through the FPI is detected using a photomultiplier tube (PMT, Philips-XP2020/Q) that is operated in photon-counting mode and read out by the data acquisition unit.

In order to measure and monitor temperature and pressure of the gas under investigation, several measurement devices are mounted within the system. The pressure is measured with a baratron, in particular an active capacitive transmitter (Pfeiffer-CMR 271) for pressure values between $100 \mathrm{hPa}$ and $1000 \mathrm{hPa}$, delivering an accuracy of $0.15 \%$ of the measured pressure value. The temperature is measured with a Pt100 thermo-resistor mounted on top of the scattering cell and delivering an accuracy of about $\pm 0.25 \mathrm{~K}$ (class A) for the temperatures measured within this study. The temperature of the gas sample is set and controlled by four Peltier elements, encased by a temperature controlled water cooling system and mounted below the scattering cell. This allows temperature settings of the gas sample from $250 \mathrm{~K}$ to $340 \mathrm{~K}$.

\subsection{Characterization of the instrument function}

The instrument function of the experiment, which is principally given by the transmission function of the FPI, has to be determined accurately to avoid systematic errors in the data retrieval. In order to do so, the FPI is illuminated with light elastically scattered from a copper wire mounted within the scattering cell which warrants that the scattered light undergoes the same collecting angle as in RB scattering from the same interaction volume. First, the free spectral range (FSR) is determined by keeping the FPI plate distance constant and scanning the laser frequency over several FSR. Subsequently, the instrument function is acquired by keeping the laser frequency constant and scanning the PZT over several FSR. After that, the nonlinearity of the piezo extension is corrected and the piezo voltage is converted into a frequency scale by exploiting that the distance between each transmission peak equals one FSR. Furthermore, the signal-to-noise ratio of the measured instrument function is increased by averaging several transmission peaks. An exemplary measurement of the instrument function is shown in Fig. 2 (black dots). The step size of the processed data is $35 \mathrm{MHz}$.

Commonly, the intensity transmission $\mathscr{T}(f)$ of an ideal FPI (i.e. axially parallel beam of rays, mirrors perfectly parallel to each other, mirrors of infinite size and mirrors without any defects) is described by an Airy function. However, in order to reach higher accuracy it is also necessary to consider defects on FPI mirrors [16]. As for instance shown in [17, 18], this can be adequately done by adding a Gaussian distributed defect term to the Airy function leading

\#221756 - \$15.00 USD Received 26 Aug 2014; revised 9 Oct 2014; accepted 9 Oct 2014; published 19 Nov 2014

(C) 2014 OSA 1 December $2014 \mid$ Vol. 22, No. 24 | DOI:10.1364/OE.22.029655 | OPTICS EXPRESS 29658 
to

$$
\mathscr{T}(f)=\frac{1}{\Gamma_{\mathrm{FSR}}}\left(1+2 \sum_{k=1}^{\infty} R^{k} \cos \left(\frac{2 \pi k f}{\Gamma_{\mathrm{FSR}}}\right) \exp \left(-\frac{2 \pi^{2} k^{2} \sigma_{g}^{2}}{\Gamma_{\mathrm{FSR}}{ }^{2}}\right)\right)
$$

where $\Gamma_{\mathrm{FSR}}$ is the FSR, $R$ is the mean mirror reflectivity and $\sigma_{g}$ is the defect parameter. It is worth mentioning that Eq. (1) without the exp-term just represents the Fourier series of the pure Airy function.

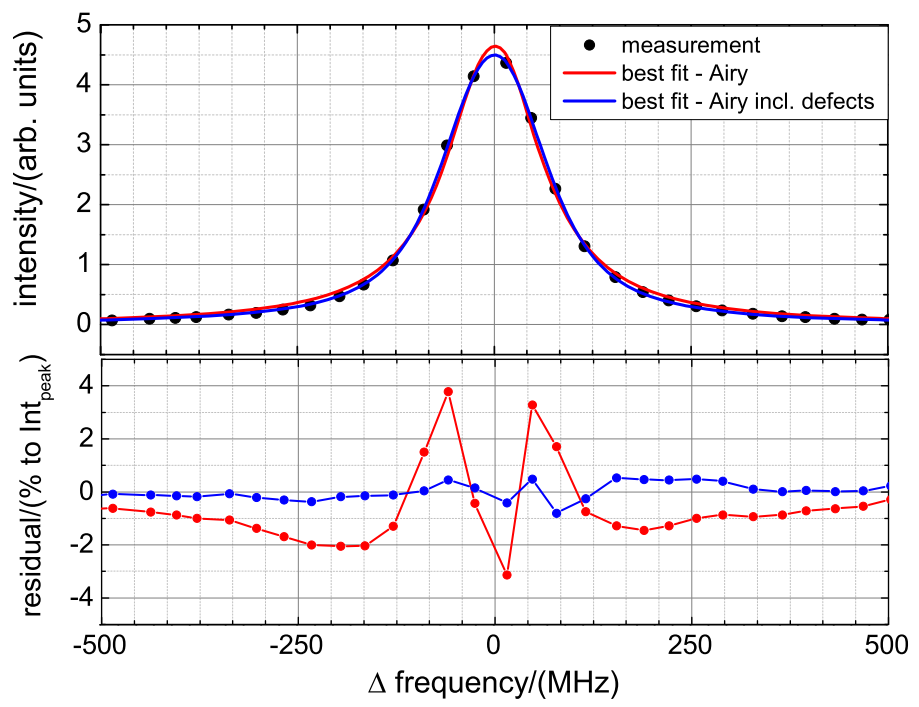

Fig. 2. (Top): Measured FPI instrument function (black dots) and best fit of an Airy function (red line) and an Airy function considering defects according to Eq. (1) (blue line). (Bottom): Respective residuals in \% with respect to the transmission peak intensity.

A least-squares fit of Eq. (1) is used to characterize the instrument function as it is shown by the blue line in Fig. 2. For comparison, the best fit of an Airy function is also indicated (Fig. 2, red line) showing deviations of $4 \%$ with respect to peak intensity, whereas the deviations are smaller than $0.5 \%$ in case defects are considered. This demonstrates the necessity of considering defects for an accurate description of the FPI transmission function. In summary, the instrument function of the system is determined by Eq. (1) with $R=0.953, \sigma_{g}=34.2 \mathrm{MHz}$ and $\Gamma_{\mathrm{FSR}}=$ $7553 \mathrm{MHz}$ leading to a full width at half maximum (FWHM) of about $146 \mathrm{MHz}$. The instrument function of the $366 \mathrm{~nm}$ experiment is different as a different FPI was used [15]. In particular, it is determined by Eq. (1) with $R=0.916, \sigma_{g}=35.7 \mathrm{MHz}$ and $\Gamma_{\mathrm{FSR}}=7440 \mathrm{MHz}$ leading to $\mathrm{FWHM} \approx 232 \mathrm{MHz}$.

\subsection{Measurement procedure for obtaining Rayleigh-Brillouin spectra}

To avoid any contamination from gases of previous measurements, the scattering cell was evacuated and flushed with air, before being charged to the desired pressure. While charging the cell, particles larger than $500 \mathrm{~nm}$ were removed by an aerosol filter in the gas inlet line. For each measurement, the gas scattering cell is charged to a designated pressure $p_{\text {amb }}$ first and sealed at room temperature $T_{\mathrm{amb}}$. Afterwards, the temperature of the cell together with the gas inside is set and controlled to a user defined value $T_{\text {meas }}$ by four Peltier elements and a temperaturecontrolled water cooling system, and measured with a Pt100 sensor. The actual pressure $p_{\text {meas }}$ of each measurement is thus different from the initial pressure and can be calculated by means

\#221756 - \$15.00 USD Received 26 Aug 2014; revised 9 Oct 2014; accepted 9 Oct 2014; published 19 Nov 2014

(C) 2014 OSA $\quad 1$ December $2014 \mid$ Vol. 22, No. 24 | DOI:10.1364/OE.22.029655 | OPTICS EXPRESS 29659 
of the ideal gas law according to

$$
p_{\text {meas }}=\frac{p_{\mathrm{amb}} \cdot T_{\text {meas }}}{T_{\mathrm{amb}}}
$$

while the number density of the gas molecules in the scattering volume stays the same.

The RB spectrum of the scattered light is resolved by changing the FPI cavity length via applying a voltage ramp to the PZT on which the curved FPI mirror is mounted. Before detecting the photons of the scattered light, the PMT is kept optically closed to determine its dark counts, which have to be subtracted from the detected signal to obtain the desired measurement data. Apart from the PMT signal and the PZT scan voltage, a signal which is proportional to the intensity of the laser inside the scattering cell is recorded with a photo diode (Fig. 1, PD) to verify that no laser power fluctuations disturb the measured spectra. After finishing the measurement, the non-linearity of the piezo-scan is corrected, and the piezo voltage is converted into a relative frequency scale by utilizing that the distance between each measured RB spectrum equals one FSR. Subsequently, several peaks (20-50) are averaged to obtain a better signal-to-noise ratio. Furthermore, as the performed light-scattering experiments do not provide an absolute intensity, the integrated intensity of the RB spectra is normalized to unity after a background correction has been performed.

Examples of RB spectra measured at $403 \mathrm{~nm}\left(T_{\text {meas }}=279.6 \mathrm{~K}, p_{\text {meas }}=870 \mathrm{hPa}\right.$ (black), $T_{\text {meas }}=296.0 \mathrm{~K}, p_{\text {meas }}=1005 \mathrm{hPa}$ (red) and $T_{\text {meas }}=330.3 \mathrm{~K}, p_{\text {meas }}=1014 \mathrm{hPa}$ (blue) $)$ and averaged for frequency intervals of $150 \mathrm{MHz}$ are shown in Fig. 3. The error bars indicate the standard deviation obtained by the averaging procedure.

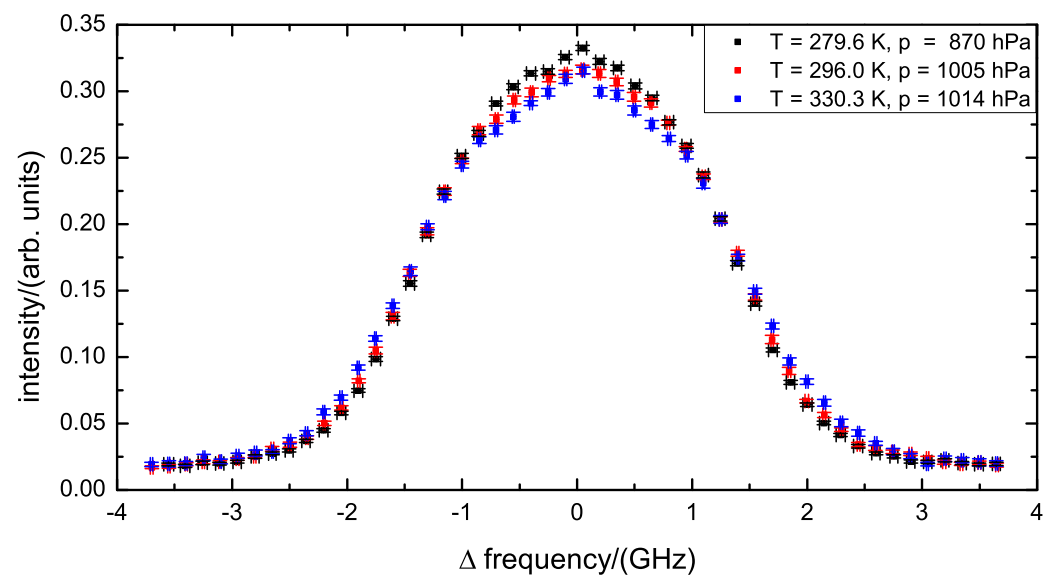

Fig. 3. RB spectra measured at $403 \mathrm{~nm}$ and different temperatures and pressures (see label), averaged for frequency intervals of $150 \mathrm{MHz}$ and normalized to equal area. The error bars indicate the standard deviation of the respective data point resulting from the averaging procedure. Details about the respective measurement conditions can be found in Table 2 .

\section{Data and data analysis}

\subsection{The temperature retrieval algorithms}

The goal of the retrieval algorithm is to obtain the temperature from measured RB spectra as accurately as possible. This is done by analyzing and comparing the measured spectra with an appropriate RB line shape model. In recent studies $[9,10,11]$ it was shown that the best

\#221756 - \$15.00 USD Received 26 Aug 2014; revised 9 Oct 2014; accepted 9 Oct 2014; published 19 Nov 2014

(C) 2014 OSA 1 December 2014 | Vol. 22, No. 24 | DOI:10.1364/OE.22.029655 | OPTICS EXPRESS 29660 
model for describing RB line shapes obtained in air is the Tenti S6 model [8]. In particular, it is shown that the deviation between model and measurement is less than $2 \%$ with respect to peak intensity. However, the Tenti S6 model appears in a mathematically complex, non-analytical closed form, which makes the application in on-line retrieval procedures for RB spectra time consuming and complicated. For that reason, Witschas $[13,14]$ developed an analytical image of the Tenti S6 model which consists of three superimposed Gaussians and which is shown to mimic the Tenti S6 model with deviations of smaller than $0.85 \%$ with respect to peak intensity for atmospherically relevant temperatures and pressures. Due to its analytical form, this line shape model can be applied to measured spectra in ordinary fit procedures.

In this study, both the Tenti S6 as well as the analytical line shape model are used for temperature retrieval to verify their level of performance. Before being applied to the measured spectra, further details are considered.

Basically, the measured line shape $\mathscr{M}$ is the convolution of the the instrument function $\mathscr{T}$ according to Eq. (1) and the spectral distribution of the scattered light $\mathscr{S}$

$$
\mathscr{M}=\mathscr{T}(f) * \mathscr{S}(T, p, f)
$$

where $*$ denotes a convolution, $f$ the optical frequency, and $T$ and $p$ the gas temperature and pressure.

In our experiments, the spectrum of the RB scattered light is mainly determined by the RB line shape $\mathscr{S}_{\mathrm{RB}}(T, p, f)$. However, it also contains an additional spectral component due to particle scattering or spurious reflections from optics and cell walls $\mathscr{S}_{\text {par }}(f)$, which appears as an additional central peak as can be seen in the measured RB spectra shown in Fig. 3. Although the particle-scattering contribution is less than $1 \%$ of the intensity of the molecular scattered light for all measurements (see also Table 2) it is necessary to consider it in order to avoid systematic errors for the temperature retrieval. As only negligible spectral broadening occurs for particle scattered or reflected light, $\mathscr{S}_{\mathrm{par}}(f)$ can be described by a Dirac-delta function $\delta_{\text {par }}$ and the spectrum of the scattered light is calculated according to

$$
\mathscr{S}(T, p, f)=I_{\mathrm{RB}} \cdot \mathscr{S}_{\mathrm{RB}}(T, p, f)+I_{\mathrm{par}} \cdot \delta_{\mathrm{par}}(f)
$$

where $I_{\mathrm{RB}}$ and $I_{\mathrm{par}}$ are the RB signal intensity and the signal intensity of light scattered on particles or spurious reflections, respectively.

Equation (3), together with Eq. (1) and Eq. (4) is now applied to the measured RB spectra in two different ways. On the one hand, $\mathscr{S}_{\mathrm{RB}}(T, p, f)$ is calculated with the Tenti S6 model [10] and considering air as an "effective" medium, consisting of molecules with an effective mass whose collisions are parametrized by effective and temperature dependent transport coefficients as summarized in Table 1.

Subsequently, Eq. (3) is numerically calculated and compared to the measured line shape for several combinations of $T$ and $I_{\mathrm{par}}$, which are the remaining two free parameters considering that $p$ is known (Eq. (2)) and $I_{\mathrm{par}}+I_{\mathrm{RB}}$ equals unity as the measured spectra are normalized to unity before they are analyzed. It is worth mentioning that even for atmospheric measurements the pressure can be taken from the standard model atmosphere as simulations show that deviations between model pressure and real pressure of $\pm 10 \mathrm{hPa}$ lead to systematic errors of less than $0.1 \mathrm{~K}$. The combination of $T$ and $I_{\text {par }}$ leading to optimum agreement between measured $\mathscr{S}_{\text {meas }}$ and modeled spectrum $\mathscr{S}_{\text {model }}$ is determined in a least-squares algorithm evaluating

$$
\chi^{2}=\sum_{i}\left(\mathscr{S}_{\text {meas }, \mathrm{i}}-\mathscr{S}_{\text {model }, \mathrm{i}}\right)^{2}
$$

The result is illustrated in Fig. 4, which shows in color-coding the calculated $\chi^{2}$ according to Eq. (5) for several $T$ and $I_{\text {par }}$ on the left, and the comparison of the best-fit spectrum with the

\#221756 - \$15.00 USD Received 26 Aug 2014; revised 9 Oct 2014; accepted 9 Oct 2014; published 19 Nov 2014 (C) 2014 OSA 1 December 2014 | Vol. 22, No. 24 | DOI:10.1364/OE.22.029655 | OPTICS EXPRESS 29661 
Table 1. Gas transport coefficients for air at temperature $T$ used for Tenti S6 model calculations [19].

\begin{tabular}{lll}
\hline Mass number & {$\left[\mathrm{g} \mathrm{mol}^{-1}\right]$} & 28.970 \\
Bulk viscosity $\eta_{b}$ & {$\left[\mathrm{~kg} \mathrm{~m}^{-1} \mathrm{~s}^{-1}\right]$} & $\eta_{b}=1.61 \cdot 10^{-7} \cdot T-3.1 \cdot 10^{-5}$ \\
Shear viscosity $\eta$ & {$\left[\mathrm{kg} \mathrm{m}^{-1} \mathrm{~s}^{-1}\right]$} & $\eta=\eta_{0} \cdot\left(\frac{T}{T_{0}}\right)^{3 / 2} \cdot \frac{T_{0}+T_{\eta}}{T+T_{\eta}}$ \\
Thermal conductivity $\kappa$ & {$\left[\mathrm{W} \mathrm{m}^{-1} \mathrm{~K}^{-1}\right]$} & $\kappa=\kappa_{0} \cdot\left(\frac{T}{T_{0}}\right)^{3 / 2} \cdot \frac{T_{0}+T_{A} \cdot \exp \left[-T_{B} / T_{0}\right]}{T+T_{A} \cdot \exp \left[-T_{B} / T\right]}$ \\
Heat capacity ratio $\gamma$ & & 1.4 \\
Internal specific heat $c_{\text {int }}$ & & 1.0 \\
\hline
\end{tabular}

Here, $\eta_{0}=1.846 \times 10^{-5} \mathrm{~kg} \mathrm{~m}^{-1} \mathrm{~s}^{-1}$ is the reference shear viscosity and $\kappa_{0}=26.24 \times 10^{-3} \mathrm{~W} \mathrm{~m}^{-1} \mathrm{~K}^{-1}$ is the reference thermal conductivity at reference temperature $T_{0}=300 \mathrm{~K} ; T_{\eta}=110.4 \mathrm{~K}, T_{A}=245.4 \mathrm{~K}$, and $T_{B}=27.6 \mathrm{~K}$ are characteristic constants for air [19].

measurement on the right (blue line). The purple area in the middle of the color-plot indicates that the value of best accordance is for $T=294.6 \mathrm{~K}$ and $I_{\mathrm{par}}=0.41 \%$ of $I_{\mathrm{RB}}$. The reference temperature was $T_{\mathrm{Pt} 100}=295.5 \mathrm{~K}$ (see also Table 2 ). On the other hand, $\mathscr{S}_{\mathrm{RB}}(T, p, f)$ is calculated by an analytical line shape model according to Witschas $[13,14]$ which is composed of the superposition of three Gaussians. Again, the contribution of particle scattering is considered by adding an additional Dirac-delta function before convolving with the instrument function as depicted in Eq. (3). Using this procedure, the measured signal can be calculated completely analytically according to

$$
\begin{aligned}
\mathscr{M}= & \frac{I_{\mathrm{RB}}}{\Gamma_{\mathrm{FSR}}} \cdot\left[\mathscr{A}\left(1+2 \sum_{k=1}^{\infty} R^{k} \cos \left(\frac{2 \pi k\left(f-f_{0}\right)}{\Gamma_{\mathrm{FSR}}}\right) \exp \left(-\frac{2 \pi^{2} k^{2}\left(\sigma_{g}{ }^{2}+\sigma_{R}^{2}\right)}{\Gamma_{\mathrm{FSR}}{ }^{2}}\right)\right)\right. \\
& +\frac{1-\mathscr{A}}{2}\left(1+2 \sum_{k=1}^{\infty} R^{k} \cos \left(\frac{2 \pi k\left(f-f_{0}-f_{B}\right)}{\Gamma_{\mathrm{FSR}}}\right) \exp \left(-\frac{2 \pi^{2} k^{2}\left(\sigma_{g}{ }^{2}+\sigma_{B}{ }^{2}\right)}{\Gamma_{\mathrm{FSR}}{ }^{2}}\right)\right) \\
& \left.+\frac{1-\mathscr{A}}{2}\left(1+2 \sum_{k=1}^{\infty} R^{k} \cos \left(\frac{2 \pi k\left(f-f_{0}+f_{B}\right)}{\Gamma_{\mathrm{FSR}}}\right) \exp \left(-\frac{2 \pi^{2} k^{2}\left(\sigma_{g}^{2}+\sigma_{B}{ }^{2}\right)}{\Gamma_{\mathrm{FSR}}{ }^{2}}\right)\right)\right] \\
+ & \frac{I_{\mathrm{par}}}{\Gamma_{\mathrm{FSR}}} \cdot\left[\left(1+2 \sum_{k=1}^{\infty} R^{k} \cos \left(\frac{2 \pi k\left(f-f_{0}\right)}{\Gamma_{\mathrm{FSR}}}\right) \exp \left(-\frac{2 \pi^{2} k^{2} \sigma_{g}{ }^{2}}{\Gamma_{\mathrm{FSR}}{ }^{2}}\right)\right)\right]
\end{aligned}
$$

where $f_{0}$ is the center frequency of the RB spectrum, and $\mathscr{A}(x, y), f_{B}(x, y), \sigma_{R}(x, y)$ and $\sigma_{B}(x, y)$ are quantities used to parameterize the line shape model and are given in $[13,14]$. $x=2 \pi f /\left(\sqrt{2} k v_{0}\right)$ and $y=p /\left(\sqrt{2} k v_{0} \eta\right)$ represent dimensionless parameters commonly used for parameterization in gas kinetic theory [8], where $k=\left|\mathbf{k}_{\mathbf{s}}-\mathbf{k}_{\mathbf{0}}\right|=4 \pi / \lambda \sin (\theta / 2)$ is the magnitude of the interacting wave vector (with $\mathbf{k}_{\mathbf{0}}$ and $\mathbf{k}_{\mathbf{s}}$ being the wave vectors of the incident and scattered light), $\lambda$ is the wavelength of the incident light, $\theta$ is the scattering angle, and $v_{0}=\left(k_{\mathrm{B}} T / m\right)^{1 / 2}$ the thermal velocity. Thus, Eq. (6), which is an analytical representation of Eq. (3), can now be applied to the measured spectra in a least-squares fit procedure, with $f_{0}$, $I_{p a r}, I_{R B}$ and $T$ (via $\mathscr{A}(x, y), f_{B}(x, y), \sigma_{R}(x, y)$ and $\sigma_{B}(x, y)$ ) being the free fit parameters and $p$ is considered to be known. An example of a best-fit of Eq. (6) to a RB line shape measurement is shown in Fig. 4, right (red dashed line). The derived temperature is $T_{\text {anal. }}=292.8 \mathrm{~K}$ and the reference temperature was $T_{\mathrm{Pt} 100}=295.5 \mathrm{~K}$.

\#221756 - \$15.00 USD Received 26 Aug 2014; revised 9 Oct 2014; accepted 9 Oct 2014; published 19 Nov 2014 (C) 2014 OSA 1 December 2014 | Vol. 22, No. 24 | DOI:10.1364/OE.22.029655 | OPTICS EXPRESS 29662 
As mentioned, the effect of particle scattering needs to be considered for both retrieval algorithms although the contribution to the signal from RB scattering is less than $1 \%$. For real atmospheric measurements, the amount of particle scattering (e.g. from the aerosol-rich boundary layer or sub-visible cirrus clouds) can be much larger. The effect of light scattering on particles and aerosols on the temperature retrieval might be systematically investigated by atmospheric high-spectral resolution lidar measurements and in future RB-scattering studies by deliberately adding aerosols at predetermined sizes and concentrations to the scattering cell and therewith mimicking their effects.
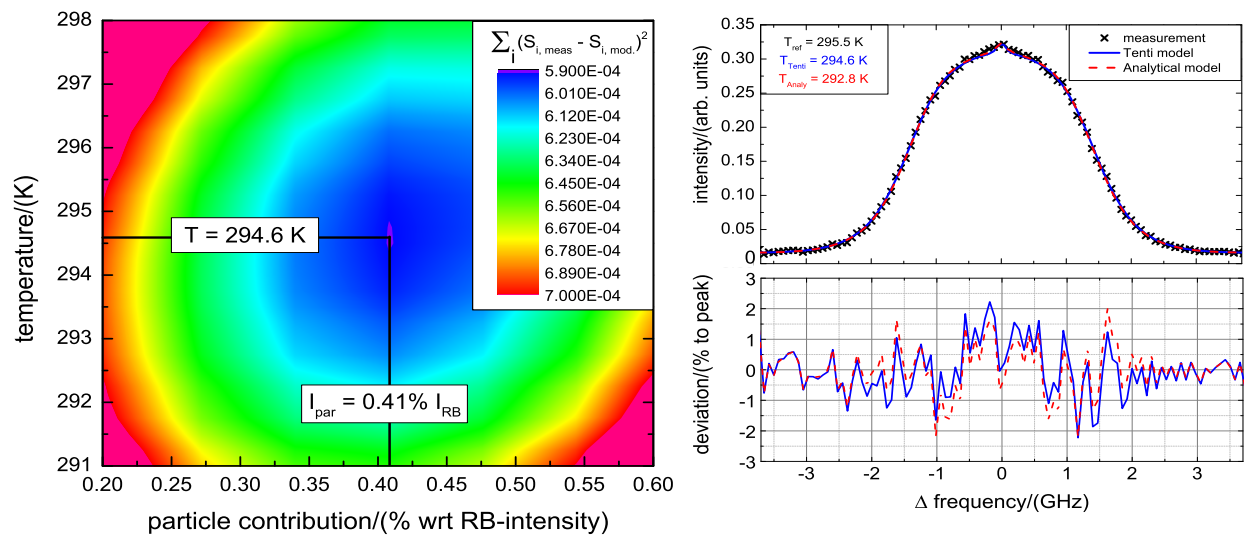

Fig. 4. (left): Squared deviation between measured and model RB line shape depending on temperature and particle concentration calculated according to Eq. (5). (right, top): Measured $\mathrm{RB}$ spectrum $\left(T_{\mathrm{Pt} 100}=295.5 \mathrm{~K}, p=1010 \mathrm{hPa}\right.$, black crosses $)$ and best-fit according to the Tenti S6 model calculation $\left(T_{\text {Tenti }}=294.6 \mathrm{~K}, I_{\mathrm{par}}=0.41 \%\right.$ of $I_{\mathrm{RB}}$, blue solid line $)$ and the analytical model calculation $\left(T_{\mathrm{anal}}=292.8 \mathrm{~K}, I_{\mathrm{par}}=0.34 \%\right.$ of $I_{\mathrm{RB}}$, red dashed line $)$. (right, bottom): Deviation between measured and model RB line shape in \% with respect to peak intensity.

\subsection{Estimation of the uncertainty of the retrieved temperature values}

In order to quantitatively evaluate the performance of the temperature retrieval algorithms, the uncertainties of the derived temperatures $T_{\text {model }}$ as well as the one of the reference temperature $T_{\mathrm{P} 100}$ have to be determined and considered.

The reference temperature $T_{\mathrm{P} t 100}$, which is considered as the actual temperature of the air inside the scattering cell, was measured with a Pt100 sensor (class A) whose permissible deviation $\Delta T_{\mathrm{Pt} 100}$ is caused by the resistance uncertainty and which is calculated according to $\Delta T_{\mathrm{Pt} 100}=0.15 \mathrm{~K}+0.002 \times\left|T_{\mathrm{Pt} 100}-273.15 \mathrm{~K}\right|$ (DIN IEC 751). Considering the temperature range of $255 \mathrm{~K}$ to $340 \mathrm{~K}$ used for the $\mathrm{RB}$ line shape measurements, $\Delta T_{\mathrm{Pt} 100}$ varies between $0.19 \mathrm{~K}$ to $0.28 \mathrm{~K}$.

For calculating the uncertainty $\Delta T_{\text {model }}$ of the retrieved temperature values $T_{\text {model }}$, two main contributors are considered within this study, namely the noise on the measured data points and the uncertainty of the scattering angle caused by the geometrical layout of the optical setup. The noise contribution is estimated by applying a maximum likelihood estimator (MLE), considering solely Poisson noise on the measured data points, and assuming for simplicity the RB spectrum to be Gaussian as it would be for very low pressures (Knudsen regime). As explicitly discussed by Hagen et al. [20], the width $w_{g}\left(w_{g}=\mathrm{FWHM} /(8 \ln 2)^{1 / 2}\right)$ of a Gaussian including 
Poisson noise can be determined with a standard deviation $\sigma_{w_{g}}=w_{g} \times(2 N)^{-1 / 2}$, where $N$ is the number of detected photons. As the RB spectrum is considered to be a Gaussian, its width is equivalently described according to $w_{g}=2 / \lambda \times\left(k_{\mathrm{B}} T / m\right)^{1 / 2}$, where $\lambda$ is the wavelength of the scattered light, $T$ is the air temperature, $k_{\mathrm{B}}$ is the Boltzmann constant, and $m$ the mass of a single air molecule. Using this relation, $\Delta T_{\text {noise }}$ is calculated to be $\Delta T_{\text {noise }}=T \times(2 / N)^{1 / 2}$ by means of the partial derivative $\left(\partial w_{g} / \partial T\right)$. Within the presented measurements, $N$ varied between $1 \times 10^{6}-5 \times 10^{6}$ photons, and $T$ was between $255 \mathrm{~K}-338 \mathrm{~K}$, thus, $\Delta T_{\text {noise }}=0.2 \mathrm{~K}-0.5 \mathrm{~K}(1-$ sigma uncertainty).

The influence of the scattering angle uncertainty can be transferred to a temperature uncertainty by means of the $x$ parameter used to parameterize RB line shapes and given before (section 3.1). Additionally, the RB spectrum is again assumed to be a Gaussian, and thus, the $y$ parameter to be zero. As the $x$ parameter depends on $\theta$ as well as on $T$, the partial derivatives $(\partial x / \partial \theta)$ and $(\partial x / \partial T)$ can be used to calculate the temperature uncertainty caused by the scattering angle according to

$$
\Delta T_{\text {angle }}=\frac{\partial \theta}{\tan (\theta / 2)} \cdot T \approx 0.02 \cdot T
$$

with $\theta=1.60 \operatorname{rad}\left(91.7^{\circ}\right)$ and $\partial \theta=0.02 \operatorname{rad}\left(1.2^{\circ}\right)$. Now, both contributions are quadratically added yielding the overall uncertainty $\Delta T_{\text {model }}$ of the retrieved temperature values as they are indicated by the error bars in Fig. 5

$$
\Delta T_{\text {model }}=\sqrt{\left(\Delta T_{\text {noise }}\right)^{2}+\left(\Delta T_{\text {angle }}\right)^{2}}
$$

It can be seen that $\Delta T_{\text {angle }}$ varies from $5.1 \mathrm{~K}$ to $6.8 \mathrm{~K}$, and thus, it is the main contributor to the overall uncertainty $\Delta T_{\text {model }}$. However, it has to be mentioned that the estimate of $\partial \theta=$ $0.02 \mathrm{rad}\left(1.2^{\circ}\right)$ is quite conservative and gives the maximum possible error. As the laser beam was only slightly re-aligned between each measurement, it is very unlikely that the principally possible scattering angle range of $\pm 1.2^{\circ}$ was exhausted. Thus, both $\partial \theta$ and with it also $\Delta T_{\text {angle }}$ are assumed to be smaller in reality. Anyway, this estimation demonstrates that RB spectra as well as the temperature retrieval from them are quite sensitive to the scattering angle itself. Regarding lidar measurements, which are usually restricted to a scattering angle of $180^{\circ}$ and a small field of view of several hundred $\mu \mathrm{rad}$, the scattering angle uncertainty will only play a minor role for a temperature retrieval from RB profiles.

\section{Experimental results and discussion}

In the following, RB line shape measurements performed at a wavelength of $403 \mathrm{~nm}$ in air ( $T=257 \mathrm{~K}$ to $330 \mathrm{~K}, p=871 \mathrm{hPa}$ to $1013 \mathrm{hPa}$ ) are used to verify the performance of the two temperature retrieval algorithms explained in section 3.1. Furthermore, previously performed RB line shape measurements ( $\lambda=366 \mathrm{~nm}, T=255 \mathrm{~K}$ to $335 \mathrm{~K}, p=643 \mathrm{hPa}$ to $826 \mathrm{hPa}$ ) published by Gu et al. [11] are used for additional verification. A summary of the respective experimental conditions for the measurements is given in Table 2.

In Fig. 5, the retrieved temperatures $T_{\text {model }}$ are plotted as a function of the reference temperature $T_{\mathrm{P} t 100}$. The left and right graph indicates the results for measurements performed at a wavelength of $366 \mathrm{~nm}$ and $403 \mathrm{~nm}$, respectively. The blue dots denote the results from the temperature retrieval using the Tenti S6 model, the red dots the results obtained by using the analytical line shape model. The shown error bars indicate the uncertainty of the reference temperature $\Delta T_{\mathrm{Pt} 100}$ and the one of the derived temperature $\Delta T_{\text {model }}$ according to Eq. (8), respectively. The gray line represents the $T_{\text {model }}=T_{\mathrm{Pt} 100}$ line.

For both data sets it is obvious that there is very good accordance between temperatures retrieved with the Tenti S6 model and reference temperature. In particular, the absolute dif-

\#221756 - \$15.00 USD Received 26 Aug 2014; revised 9 Oct 2014; accepted 9 Oct 2014; published 19 Nov 2014

(C) 2014 OSA 1 December 2014 | Vol. 22, No. 24 | DOI:10.1364/OE.22.029655 | OPTICS EXPRESS 29664 
ference is less than $2 \mathrm{~K}$ for all measurements. A linear correlation of retrieved- and reference temperature (Fig. 5, blue dashed lines) yields a slope of $1.00 \pm 0.03(\lambda=366 \mathrm{~nm})$ and $1.01 \pm 0.02(\lambda=403 \mathrm{~nm})$, and thus clearly demonstrates the reliability of the retrieval procedure. The second algorithm using the analytical line shape model shows larger discrepancies of up to $9.9 \mathrm{~K}$ and is thus not useful at its present stage. The linear correlation of retrieved- and reference temperature (Fig. 5, red dashed lines) yields a slope of $0.89 \pm 0.03(\lambda=366 \mathrm{~nm})$ and $0.87 \pm 0.02(\lambda=403 \mathrm{~nm})$, and thus indicates that the model leads to larger discrepancies at higher temperatures. The statistical uncertainty of the retrieved temperature values varies between $5 \mathrm{~K}$ and $7 \mathrm{~K}$. However, it is worth mentioning that the uncertainty caused by Poisson noise only varies between $0.2 \mathrm{~K}$ and $0.5 \mathrm{~K}$. The larger contribution comes from the scattering angle uncertainty associated with the present experimental setup. As already explained, this estimation is conservative and gives the maximum uncertainty that can be caused due to a varying scattering angle. Considering that lidar measurements have scattering angles of $180^{\circ}$, only the Poisson noise uncertainty $(0.2 \mathrm{~K}$ to $0.5 \mathrm{~K})$ would contribute to such kind of atmospheric measurements.
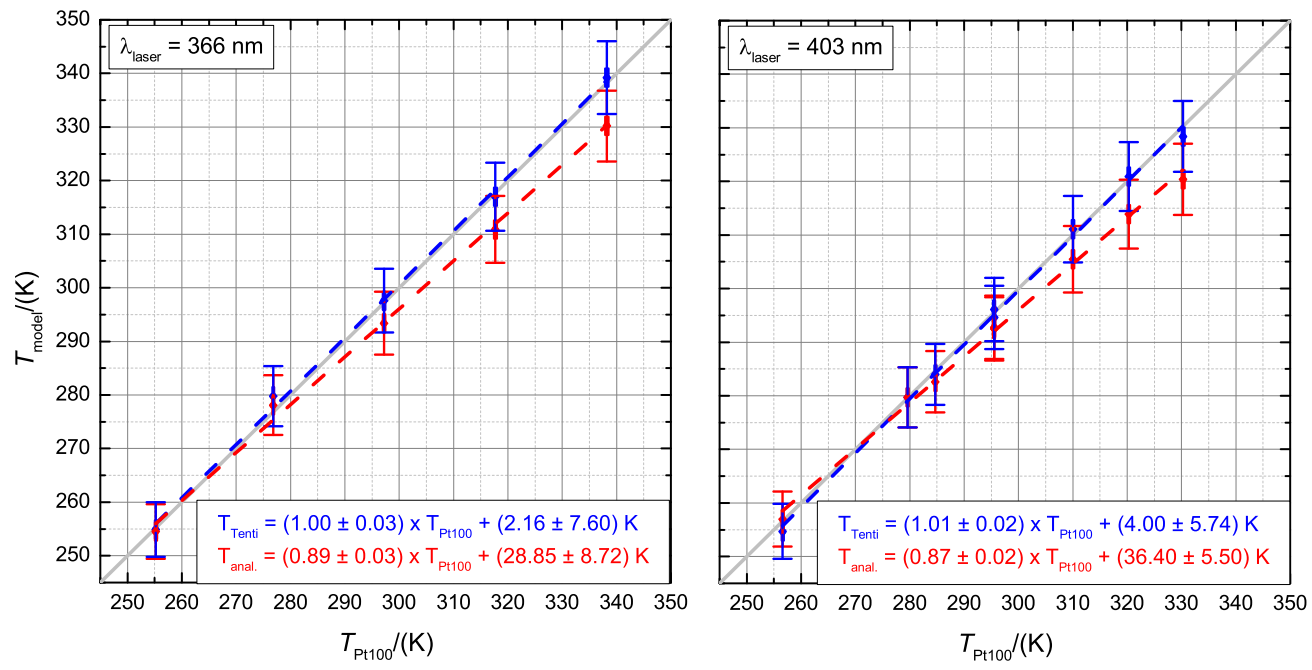

Fig. 5. Temperature values retrieved from RB spectra measured at $366 \mathrm{~nm}$ (left) and $403 \mathrm{~nm}$ (right) by using the Tenti S6 model (blue) and the analytical line shape model (red) compared to reference temperature measured with a Pt100 sensor. Detailed values are given in Table 2. The gray line indicates $T_{\text {model }}=T_{\mathrm{Pt} 100}$ line.

The results shown above demonstrate that absolute temperatures can be derived from RB spectra obtained in air with an accuracy better than $2 \mathrm{~K}$ by utilizing the described temperature retrieval based on the Tenti S6 model. The retrieval based on the analytical model leads to larger deviations especially at higher temperatures. This behavior can be explained by a poor temperature-parameterization method within the analytical line shape model [13, 14]. In particular, the analytical model is parameterized by the $x$ - and $y$ parameter which themselves depend on pressure and temperature. However, in order to obtain different line shapes with different $x$ and $y$ parameters for the parameterization process, the temperature was kept constant at $250 \mathrm{~K}$ and only the pressure values have been varied. Thus, the entire model is actually only valid for a temperature of $T=250 \mathrm{~K}$. No temperature dependency of the gas transport coefficients (see Table 1) is considered. This explanation is also confirmed by the derived values at low temperatures which are $T_{\text {anal. }}=257.0 \mathrm{~K}$ at a reference temperature of $T_{\mathrm{P} t 100}=256.6 \mathrm{~K}$ (at

\#221756 - \$15.00 USD Received 26 Aug 2014; revised 9 Oct 2014; accepted 9 Oct 2014; published 19 Nov 2014 (C) 2014 OSA 1 December 2014 | Vol. 22, No. 24 | DOI:10.1364/OE.22.029655 | OPTICS EXPRESS 29665 
$403 \mathrm{~nm}$ ) and $T_{\text {anal. }}=254.5 \mathrm{~K}$ at a reference temperature of $T_{\mathrm{P} 100}=255.2 \mathrm{~K}$ (at $366 \mathrm{~nm}$ ). In both cases the deviation between derived- and reference temperature is less than $1 \mathrm{~K}$. This means that the analytical model needs further development, in particular, the consideration of different temperature values before it is useful for realistic temperature retrievals. For an improvement concerning the parameterization process it is foreseen to utilize several Tenti S6 line shapes calculated for different wavelengths, scattering angles and temperature- and pressure values in order to find improved values for the parameters needed to evaluate Eq. (6). In case that this approach leads not to an improvement, a new approach can be explored based on deriving the analytical model for a fixed wavelength and scattering angle, just depending on temperature and pressure.

As an alternative, an analytical RB line shape model, recently developed by Ma et al. [21, 22] and based on the superposition of three Voigt-functions, was shown to deliver improved performance in temperature retrieval over the three-Gaussian model, especially at pressure values larger than $1000 \mathrm{hPa}$ [21]. The temperature in this V3-model are directly derived from the Brillouin shift as it is usually done with RB spectra measured in the hydrodynamic regime (e.g. in gases with pressures larger than 5 bar, liquids or solid states). However, as discussed in [21], the temperature derived from a simulated reference RB spectrum at a pressure of $1000 \mathrm{hPa}$ and temperature of $292 \mathrm{~K}$ has an offset of $8.4 \%$ with respect to the reference value. Thus, also this V3 analytical model needs further improvement before it can be used for temperature retrievals from RB spectra at atmospheric conditions.

In case it turns out that analytical models do not deliver the desired accuracy for the temperature retrieval, a Tenti S6 line shape based look-up table might be used to end up with a faster processing time while keeping the accuracy demonstrated here.

Table 2. Overview of experimental conditions and retrieved temperature values.

\begin{tabular}{ccccccccc}
\hline $\begin{array}{c}T_{\mathrm{P} 100} \\
(\mathrm{~K})\end{array}$ & $\begin{array}{c}T_{\text {Tenti }} \\
(\mathrm{K})\end{array}$ & $\begin{array}{c}I_{\text {par,Tenti }} \\
(\%)\end{array}$ & $\begin{array}{c}T_{\mathrm{Pt} 100}-T_{\text {Tenti }} \\
(\mathrm{K})\end{array}$ & $\begin{array}{c}T_{\text {anal. }} \\
(\mathrm{K})\end{array}$ & $\begin{array}{c}I_{\text {par,anal. }} \\
(\%)\end{array}$ & $\begin{array}{c}T_{\mathrm{Pt} 100}-T_{\text {anal. }} \\
(\mathrm{K})\end{array}$ & $\begin{array}{c}p \\
(\mathrm{hPa})\end{array}$ & $\begin{array}{c}\lambda \\
(\mathrm{nm})\end{array}$ \\
\hline 296.0 & 296.1 & 0.66 & -0.1 & 292.5 & 0.68 & +3.5 & 1005 & 402.987 \\
256.6 & 254.7 & 0.41 & +1.9 & 257.0 & 0.35 & -0.4 & 880 & 402.995 \\
295.5 & 294.6 & 0.41 & +0.9 & 292.8 & 0.34 & +2.7 & 1010 & 402.985 \\
309.2 & 311.1 & 0.41 & -1.9 & 305.5 & 0.38 & +3.7 & 1011 & 402.995 \\
330.3 & 328.4 & 0.75 & +1.9 & 320.4 & 0.82 & +9.9 & 1014 & 402.993 \\
284.7 & 284.0 & 0.34 & +0.7 & 282.6 & 0.41 & +2.1 & 955 & 402.997 \\
279.6 & 279.7 & 0.48 & -0.1 & 279.7 & 0.43 & -0.1 & 870 & 402.997 \\
320.3 & 320.9 & 0.27 & -0.6 & 313.9 & 0.24 & +6.4 & 1013 & 402.996 \\
\hline 255.2 & 254.9 & 0.34 & +0.3 & 254.5 & 0.16 & +0.7 & 643 & 366.840 \\
276.8 & 278.6 & 0.34 & -1.8 & 278.1 & 0.27 & -1.3 & 703 & 366.840 \\
297.2 & 297.6 & 0.48 & -0.4 & 293.4 & 0.42 & +3.8 & 726 & 366.650 \\
317.7 & 317.0 & 0.41 & +0.7 & 310.9 & 0.46 & +6.8 & 776 & 366.650 \\
338.2 & 339.1 & 0.55 & -0.9 & 330.2 & 0.62 & +8.0 & 826 & 366.650 \\
\hline
\end{tabular}

\section{Summary and conclusion}

Rayleigh-Brillouin (RB) scattering measurements in air $(\lambda=403 \mathrm{~nm}, T=257 \mathrm{~K}$ to $330 \mathrm{~K}$, $p=871 \mathrm{hPa}$ to $1013 \mathrm{hPa}$ ) were performed and used to verify the performance of two different temperature retrieval algorithms, one based on the Tenti S6 line shape model [8], and one based on an analytical model $[13,14]$. Furthermore, previously performed RB measurements (air, $\lambda=$ $366 \mathrm{~nm}, T=255 \mathrm{~K}$ to $335 \mathrm{~K}, p=643 \mathrm{hPa}$ to $826 \mathrm{hPa}$ ) [11] were used for additional validation.

\#221756 - \$15.00 USD Received 26 Aug 2014; revised 9 Oct 2014; accepted 9 Oct 2014; published 19 Nov 2014 (C) 2014 OSA 1 December $2014 \mid$ Vol. 22, No. 24 | DOI:10.1364/OE.22.029655 | OPTICS EXPRESS 29666 
With both data sets it is demonstrated that absolute temperature can be derived from RB spectra obtained in air at atmospheric conditions with high accuracy. In particular, it is shown that the accordance of the derived temperature to the reference temperature is better than $2 \mathrm{~K}$ in case of the Tenti S6 model-based retrieval algorithm. This outcome is of great relevance for future high spectral resolution lidar systems that might use RB spectra for deriving atmospheric temperature profiles as it was recently shown by Witschas et al. [23]. The retrieval based on the analytical model leads to discrepancies between retrieved- and reference temperature of up to $9.9 \mathrm{~K}$ and is thus not useful at its present stage. It is discussed that these discrepancies are explained by a poor temperature-parameterization within the model which has to be improved for successful future use.

The present high-quality experimental data demonstrate that temperatures can be retrieved from RB line shapes at an accuracy of $2 \mathrm{~K}$. It is worth mentioning that this accuracy is depending on the instrument resolution and signal-to-noise ratio of the measured RB spectra. Thus, this has to be considered when estimating the accuracy for realistic atmospheric lidar measurements. Also the effect of aerosol scattering will be decisive under real atmosphere conditions.

While the present study has shown that the Tenti S6 model best describes the line shapes in RB-scattering, future studies should reveal how accurate temperature retrieval procedures can become for certain values of resolution, signal-to-noise ratio and aerosol contributions.

\section{Acknowledgments}

We thank A.G. Straume (ESA), O. Reitebuch, C. Lemmerz and P. Vrancken (DLR), and M.O. Vieitez (KNMI) for fruitful discussions, and J. Busz (De Haagse School) for help with the experimental setup. The valuable comments of two anonymous reviewers are highly appreciated. This work was partly funded by DLR and ESA within the Network Partnering Initiative, contract No. 4000104130 and by ESA, contract No. 21396. Furthermore, the research leading to these results has received funding from LASERLAB-EUROPE (grant agreement No. 284464, EC's Seventh Framework Programme). The core part of the code that computes the Tenti S6 model has been kindly provided to us by X. Pan and W. van de Water.

\#221756 - \$15.00 USD Received 26 Aug 2014; revised 9 Oct 2014; accepted 9 Oct 2014; published 19 Nov 2014

(C) 2014 OSA 1 December 2014 | Vol. 22, No. 24 | DOI:10.1364/OE.22.029655 | OPTICS EXPRESS 29667 